\title{
Durable Response to Osimertinib in a Chinese Patient with Metastatic Lung Adenocarcinoma Harboring a Rare EGFR L858R/D76IY Compound Mutation
}

This article was published in the following Dove Press journal: OncoTargets and Therapy

Yajie Zhu',*

Jianning Tang ${ }^{1} *$

Xin $\mathrm{Li}^{1}$

Tian Qin ${ }^{2}$

Yang Wei ${ }^{1}$

'Department of Medical Oncology, Sichuan Cancer Hospital \& Institute, Sichuan Cancer Center, School of Medicine, University of Electronic Science and Technology of China, Chengdu 6I004I, People's Republic of China; ${ }^{2}$ Burning Rock Biotech, Guangzhou 510300, People's Republic of China

*These authors contributed equally to this work
Correspondence: Yang Wei

Department of Medical Oncology, Sichuan Cancer Hospital \& Institute, Sichuan

Cancer Center, School of Medicine,

University of Electronic Science and

Technology of China, No. 55, Section 4, South Renmin Road, Chengdu 61004I,

People's Republic of China

Tel +86-28-85420243

Email 2224326936@qq.com
Abstract: Uncommon mutations account for $10-15 \%$ of epidermal growth factor receptor $(E G F R)$ mutations in patients with non-small-cell lung cancer (NSCLC). However, in spite of the wealth of knowledge of the clinical significance and tyrosine kinase inhibitor (TKI) sensitivity of these mutations, acquisition of deeper insights is limited by the paucity of case reports and cohort studies of the exceptionally rare mutations, including compound mutations. In the present case, we describe the clinical efficacy of icotinib and osimertinib in a metastatic lung adenocarcinoma patient carrying a highly uncommon EGFR L858R/D761Y compound mutation. The progression-free survival (PFS) with osimertinib treatment was much longer than that with icotinib (19 mo vs $8.2 \mathrm{mo}$ ), and the overall survival (OS) has currently exceeded three years. To the best of our knowledge, this is the first report of durable osimertinib response in an NSCLC patient with a rare EGFR L858R/D761Y mutation.

Keywords: NSCLC, EGFR, compound mutation, icotinib, osimertinib, D761Y

\section{Introduction}

With an incidence of $39.6 \%$ in Asian Pacific patients, mutations in epidermal growth factor receptor $(E G F R)$ constitute a major tumorigenic mechanism in nonsmall-cell lung cancer (NSCLC). ${ }^{1}$ The most common actionable ones account for $80-90 \%$ of all EGFR mutations. ${ }^{2}$ These therapeutically relevant mutations, including the exon 19 deletion and the L858R mutation in exon 21, are mostly targets of tyrosine kinase inhibitors (TKIs). The remaining 10-20\% uncommon mutations make up a heterogeneous group of single (ie, G719X, S768I, L861Q) and compound gene alterations within exons $18-21 .^{3}$ In recent years, a number of studies have reported the TKI sensitivity and clinical significance of EGFR compound mutations. ${ }^{4}$ Here, we present a case of advanced NSCLC harboring an EGFR L858R/D761Y compound mutation, in which osimertinib elicited a more durable response following progression on initial icotinib treatment.

\section{Case Report}

A 62-year-old female nonsmoker presented to our hospital with a two-month history of back pain. Contrast-enhanced computed tomography (CT) scan showed 
bone metastasis in the thoracolumbar vertebra (T4-5, L2), causing the back pain. Incidentally, a mass on the upper left lobe of lung was found along with mediastinal adenopathy. Histopathology review of the pulmonary mass suggested invasive adenocarcinoma. Next generation sequencing (NGS) was performed on circulating tumor DNA (ctDNA) to profile the mutation status of 12 genes associated with known oncogenic driver mutations in NSCLC (EGFR, ALK, HER2, BRAF, KRAS, MET, ROS1, RET, NTRK1, NTRK2, NTRK3 and PIK3CA). Only two mutations were found, namely EGFR-L858R in exon 21 and D761Y in exon 19 (allelic frequency, AF: 4.34\%, Figure 1). The patient was accordingly treated with firstgeneration EGFR-TKI icotinib (150 mg, po, tid). Follow- up CT scans after three months revealed slightly decreased primary lesion and mediastinal lymph nodes compared with previous images. The efficacy of the first-line icotinib treatment was assessed as stable disease (SD) per RECIST version 1.1. After continued icotinib administration for another 6 months, the patient developed severe thoracalgia and dyspnea. Chest CT scans revealed a marked increase in tumor size that led to a re-evaluation of progressive disease. The PFS of icotinib was therefore 8.2 months (Figures 2 and 3).

NGS was performed again to examine the status of EGFR T790M and other mutations known to lead to icotinib resistance, but the genomic alterations were same as before and the AF was increased to $5.25 \%$. Upon informed

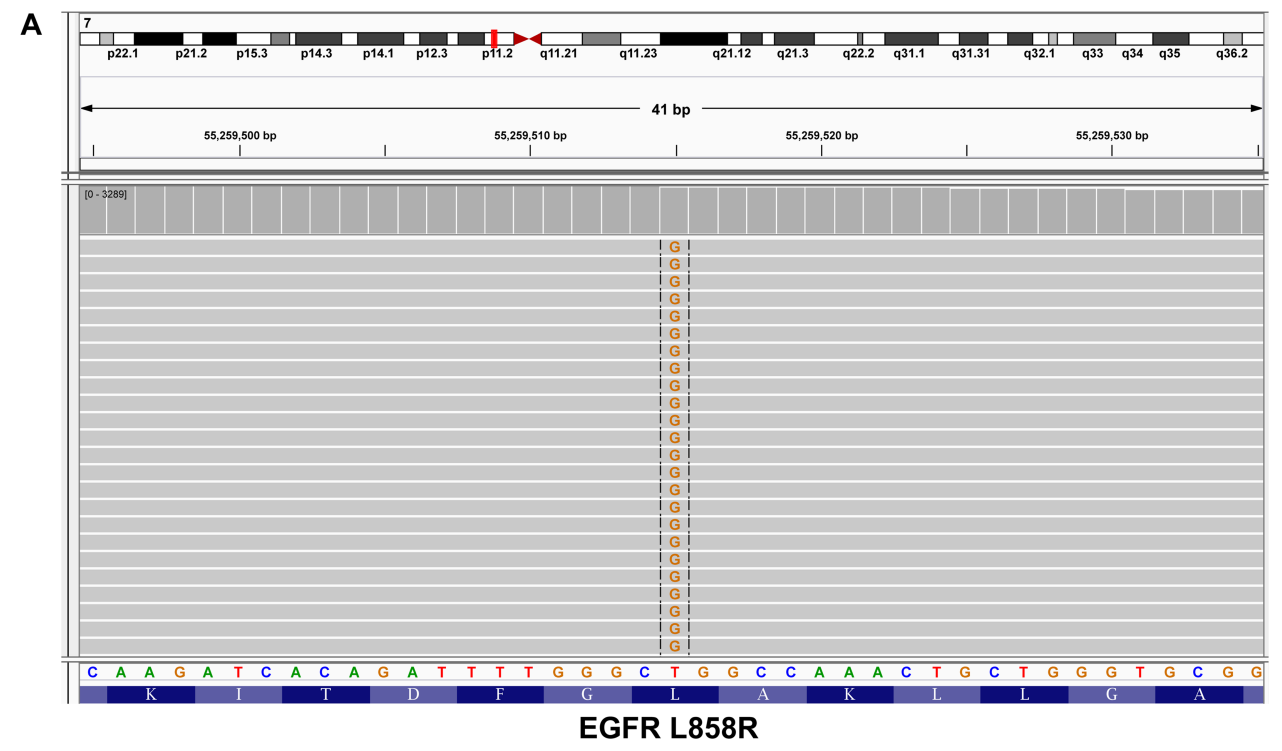

B

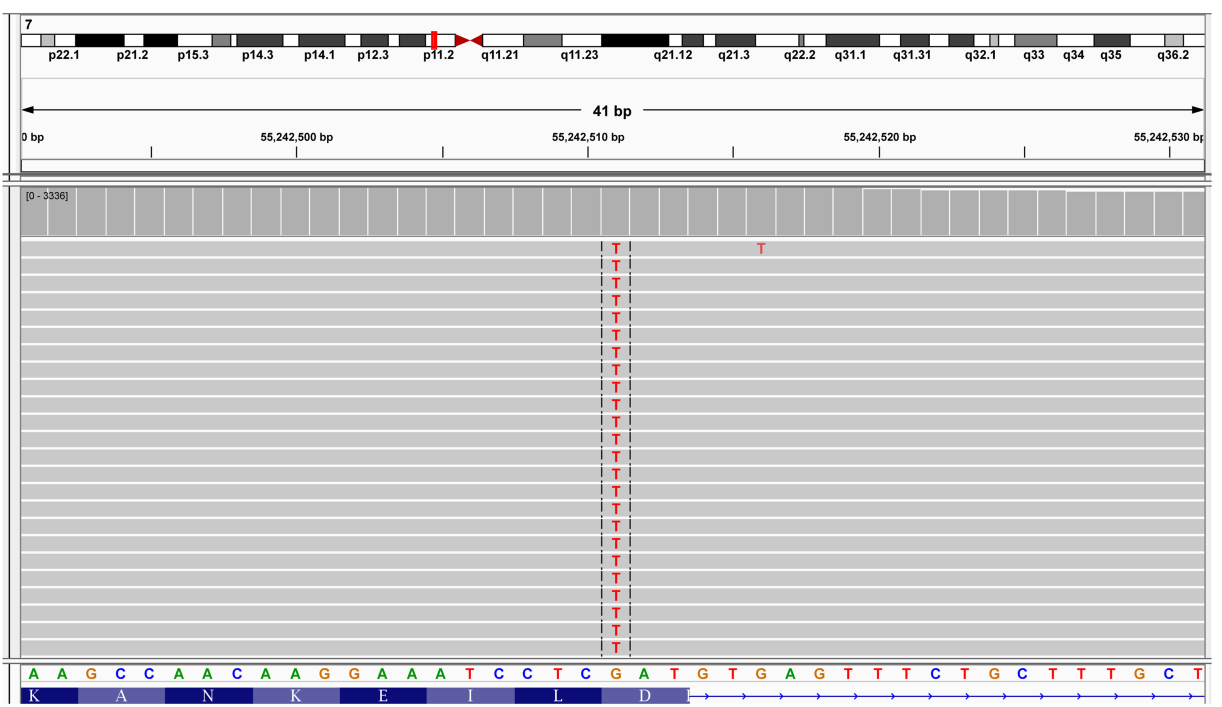

EGFR D761Y

Figure I Next-generation sequencing identified an EGFR compound mutation consisting of L858R in exon 2 I (A) and D76IY in exon I9 (B) from plasma. 
March 25, 2017

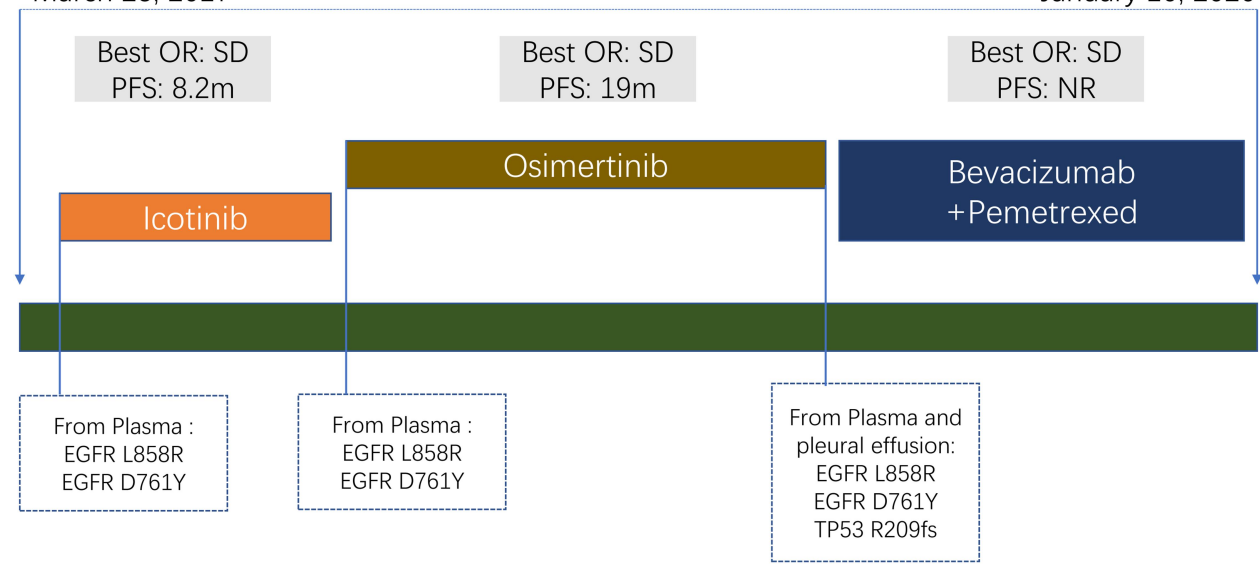

Figure 2 Diagram of the course of disease management, showing different treatment regimens prescribed and the results of mutation analyses.

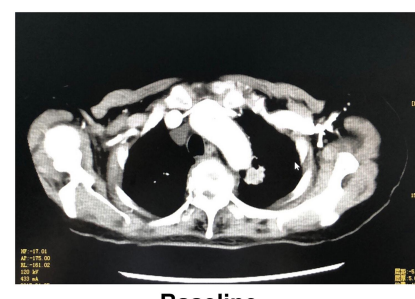

Baseline

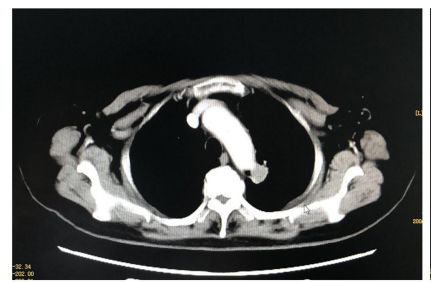

SD at $11 \mathrm{~m}$ osimertinib

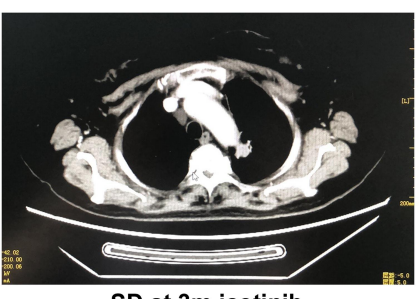

SD at $3 \mathrm{~m}$ icotinib

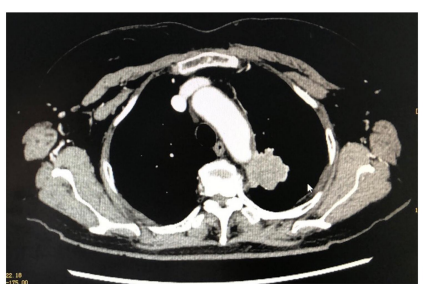

PD after $19 \mathrm{~m}$ of osimertinib

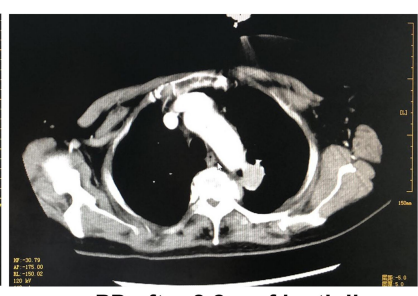

PD after $8.2 \mathrm{~m}$ of icotinib

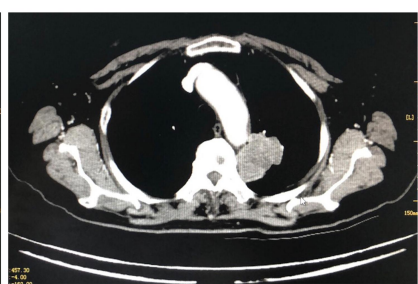

SD at $3 \mathrm{~m}$ chemotherapy

Figure 3 Representative CT images at indicated time points, shown along with corresponding treatment regimen and efficacy evaluation.

consent, osimertinib was administered at $80 \mathrm{mg}$ once daily accompanied with zoledronic acid injection for bone metastases. CT scans 11 months later showed decreased primary pulmonary lesion. The best response of osimertinib treatment was SD and the PFS was 19 months (Figures 2 and 3). Both pleural effusion and plasma ctDNA were subjected to capture-based ultra-deep sequencing that target the exons and critical introns of 168 cancer-related genes (LungPlasma ${ }^{\mathrm{TM}}$, Burning Rock Biotech, China). In addition to the two EGFR mutations detected the previous sequencing tests (AF: $5.76 \%$ in pleural effusion and $4.34 \%$ in plasma), a mutation in TP53 R209fs was identified (AF: $2.52 \%$ in pleural effusion and $1.88 \%$ in plasma). Chemotherapy with bevacizumab plus pemetrexed was prescribed as third-line therapy as per standard of care. On the latest follow-up in April, 2020, neither of the primary and metastatic lesions presented notable change in size (Figures 2 and 3). There were no significant adverse events throughout the course of treatment. An informed consent was obtained for the publication of the case report.

\section{Discussion}

This case reported the clinical efficacy of icotinib and osimertinib in a patient with metastatic NSCLC carrying an EGFR L858R/D761Y double mutation. The PFS with osimertinib treatment is much longer than that with icotinib (19 mo vs $8.2 \mathrm{mo}$ ) and the OS is more than 3 years as of the time of manuscript preparation.

$E G F R \mathrm{D} 761 \mathrm{Y}$ is an exceptionally rare mutation in lung cancer and other solid tumors. Previous research has suggested an association between D761Y and acquired resistance to EGFR-TKI, but suffers from lack of evidence due to paucity of relevant cases. ${ }^{5}$ Marissa et al reported about 
an NSCLC patient with an OS of 13 months on gefitinib treatment. EGFR D761Y mutation was identified upon autopsy in a brain metastasis, suggesting a potential association between this mutation and resistance to gefitinib. Cell viability assays confirmed this association in vitro, where sensitivity to EGFR-TKIs decreased in cells harboring L858R/D761Y mutation. ${ }^{6}$ Two other investigations further reported the role of $\mathrm{D} 761 \mathrm{Y}$ in not only acquired but also primary resistance to gefitinib. ${ }^{7,8}$ Interestingly, an in vitro study has showed that despite displaying resistance to first-generation reversible EGFR-TKIs, L858R/D761Ymutant cells were sensitive to irreversible inhibitors, especially the third-generation EGFR-TKIs. ${ }^{9}$ In this case, we reported about a lung adenocarcinoma patient carrying L858R/D761Y double mutation and showing more favorable response to osimertinib than to icotinib, thereby providing clinical evidence supporting the in vitro findings. To the best of our knowledge, this is the first report of durable osimertinib response in NSCLC patient with an EGFR L858R/D761Y compound mutation.

Icotinib is a highly specific and selective reversible EGFR-TKI with proven noninferior efficacy to gefitinib as second-/third-line treatment in patients with advanced NSCLC, as well as superior efficacy as first-line treatment when compared with chemotherapy in treating EGFRmutant NSCLC. ${ }^{10,11}$ A recent clinical trial has demonstrated that high-dose icotinib improved median PFS and objective response rate (ORR) in patients with NSCLC harboring EGFR L858R mutation with acceptable tolerability. $^{12}$ In our patient, icotinib at standard dosage showed an acceptable efficacy in treating L858R/D761Ymutant lung adenocarcinoma, although the response was less beneficial than that to osimertinib. The underlying molecular mechanisms remain to be elucidated, but could hinge on the location of the D761Y mutation site. The EGFR tyrosine kinase domain is comprised of an N-terminal lobe, a C-terminal lobe, and within the cleft of the two lobes an active ATP-binding site, which is vulnerable competitive binding with reversible EGFRTKIs. Substitution of the Lys 858 amino acid residue with an arginine could reduce the affinity for ATP and the competition for binding sites, leading to an enhanced receptor dimerization and activity. ${ }^{2}$ The $\mathrm{D} 761 \mathrm{Y}$ mutation (2281G $>$ T in the DNA sequence) is located on the edge of exon 19 and predicted to be part of the $\alpha$-C-helix adjacent to the ATP-binding site. ${ }^{6}$ It is therefore conceivable that this mutation may negatively regulate the binding affinity to reversible EGFR-TKIs. In this scenario, ATP- independent, irreversible TKIs would elicit more favorable clinical outcomes, as their affinity to the ATP-binding site remains unaffected by the D761Y mutation. Further biochemical and cellular studies are warranted to test this hypothesis.

In conclusion, we present a case of an exceptionally rare compound EGFR L858R/D761Y mutation in a patient with metastatic lung adenocarcinoma treated serially with icotinib and osimertinib monotherapies. Notably, this is to our knowledge the first report of durable osimertinib response in treating advanced NSCLC carrying a double L858R/D761Y mutation in EGFR. Since clinical trials are not likely due to the scarcity of this compound mutation, single case reports and prospective case series, along with biochemical characterizations, are all the more important in establishing an evidenced-based treatment strategy for treating patients carrying this highly uncommon mutation.

\section{Ethical Approval}

Institutional approval was not required to publish the case details.

\section{Patient Informed Consent}

Written informed consent was obtained from the patient for the publication of his case details and images.

\section{Acknowledgment}

We are grateful to the patient and her family. Also, we wish to express our gratitude to the clinical investigators, study coordinators, operation staff, and all other team members on this project.

\section{Disclosure}

Tian Qin is an employee of Burning Rock Biotech. The authors declare no other potential conflicts of interest for this work. The first two authors contributed equally and should be regarded as co-first authors.

\section{References}

1. Yatabe Y, Kerr KM, Utomo A, et al. EGFR mutation testing practices within the Asia Pacific region: results of a multicenter diagnostic survey. $J$ Thorac Oncol. 2015;10(3):438-445. doi:10.1097/ JTO.0000000000000422

2. Li WQ, Cui JW. Non-small cell lung cancer patients with ex19del or exon 21 L858R mutation: distinct mechanisms, different efficacies to treatments. J Cancer Res Clin Oncol. 2020;146(9):2329-2338. doi:10.1007/s00432-020-03296-6

3. Graham RP, Treece AL, Lindeman NI, et al. Worldwide frequency of commonly detected EGFR mutations. Arch Pathol Lab Med. 2018;142 (2):163-167. doi:10.5858/arpa.2016-0579-CP 
4. Yu X, Zhang X, Zhang Z, et al. First-generation EGFR tyrosine kinase inhibitor therapy in 106 patients with compound EGFR-mutated lung cancer: a single institution's clinical practice experience. Cancer Commun. 2018;38(1):51. doi:10.1186/s40880-018-0321-0

5. Nguyen KS, Kobayashi S, Costa DB. Acquired resistance to epidermal growth factor receptor tyrosine kinase inhibitors in non-small-cell lung cancers dependent on the epidermal growth factor receptor pathway. Clin Lung Cancer. 2009;10(4):281-289. doi:10.3816/CLC.2009.n.039

6. Balak MN, Gong Y, Riely GJ, et al. Novel D761Y and common secondary T790M mutations in epidermal growth factor receptor-mutant lung adenocarcinomas with acquired resistance to kinase inhibitors. Clin Cancer Res. 2006;12(21):6494-6501. doi:10.1158/1078-0432.CCR-06-1570

7. Toyooka S, Date H, Uchida A, Kiura K, Takata M. The epidermal growth factor receptor D761Y mutation and effect of tyrosine kinase inhibitor. Clin Cancer Res. 2007;13(11):3431; author reply 3431-3432. doi:10.1158/1078-0432.CCR-07-0070

8. Tokumo M, Toyooka S, Ichihara S, et al. Double mutation and gene copy number of EGFR in gefitinib refractory non-small-cell lung cancer. Lung Cancer. 2006;53(1):117-121. doi:10.1016/j.lungcan.2006.04.008
9. Chiba M, Togashi Y, Bannno E, et al. Efficacy of irreversible EGFRTKIs for the uncommon secondary resistant EGFR mutations L747S, D761Y, and T854A. BMC Cancer. 2017;17(1):281. doi:10.1186/ s12885-017-3263-Z

10. Shi Y, Zhang L, Liu X, et al.Icotinib versus gefitinib in previously treated advanced non-small-cell lung cancer (ICOGEN): a randomised, double-blind phase 3 non-inferiority trial. Lancet Oncol. 2013;14(10):953-961. doi:10.1016/S1470-2045(13)70355-3

11. Shi YK, Wang L, Han BH, et al. First-line icotinib versus cisplatin/ pemetrexed plus pemetrexed maintenance therapy for patients with advanced EGFR mutation-positive lung adenocarcinoma (CONVINCE): a Phase 3, open-label, randomized study. Ann Oncol. 2017;28(10):2443-2450.

12. Li X, Zhang L, Jiang D, et al. Routine-dose and high-dose icotinib in patients with advanced non-small cell lung cancer harboring EGFR exon 21-1858r mutation: the randomized, phase II, INCREASE Trial. Clin Cancer Res. 2020;26(13):3162-3171. doi:10.1158/1078-0432. CCR-19-3064

\section{Publish your work in this journal}

OncoTargets and Therapy is an international, peer-reviewed, open access journal focusing on the pathological basis of all cancers, potential targets for therapy and treatment protocols employed to improve the management of cancer patients. The journal also focuses on the impact of management programs and new therapeutic agents and protocols on patient perspectives such as quality of life, adherence and satisfaction. The manuscript management system is completely online and includes a very quick and fair peer-review system, which is all easy to use. Visit http://www.dovepress.com/ testimonials.php to read real quotes from published authors. 